Elmar Mittler*

\title{
Transfer und Transformation
}

\author{
Schlaglichter auf die Entwicklung der Bibliotheken in den letzten 50 Jahren und auf ihre \\ Zukunft
}

https://doi.org/10.1515/bfp-2021-0022

Zusammenfassung: Es werden wichtige Etappen der Bibliotheksentwicklung der letzten 50 Jahre angesprochen. Am Anfang stehen die Bibliothekspläne der 70er- und 80er-Jahre, die Grundlagen oder Forderungskataloge für den Ausbau und die Reorganisation des Bibliothekswesens waren und nach der Wiedervereinigung die schnelle Integration der Bibliotheken in den neuen Ländern befördert haben. Die Ausstellung Bibliotheca Palatina 1986 mit ihrem überwältigenden Publikumserfolg war Ausgangspunkt der Wiedergewinnung der ehemals Heidelberger Bibliothekschätze durch Mikroverfilmung, Digitalisierung und Forschung. Die EDV-Entwicklung führte zunächst zur gläsernen Bibliothek in weltweiter Vernetzung. Inzwischen ist eine Metamorphose der Bibliotheken im Gang, die ihre Bestände virtuell grenzenlos zur Verfügung stellen können und in ihren Gebäuden neben Informationsmedien aller Art auch Raum für den Wissenserwerb durch aktives, oft kollaboratives Gestalten anbieten. So werden die Bibliotheken - unterstützt durch funktionale und oft auch faszinierende Architektur - immer mehr von den Bibliothekarsbibliotheken der Vergangenheit zu den Nutzerbibliotheken von morgen.

Schlüsselwörter: Bibliotheksplanung; Bibliotheca Palatina; Bibliotheksarchitektur; Bibliotheksautomatisierung; Nutzerbibliothek

\section{Transfer and Transformation Hotspots of the Development of the Libraries in the Last 50 Years and Their Future}

Abstract: The main milestones of library development of the last 50 years are on the focus. Library plans of the 70es and 80es are the basic concepts and checklists for development and reorganisation of libraries and alleviated the integration of the libraries in the new states after the reunion. The exhibition „Bibliotheca Palatina“ 1986 was

*Kontaktperson: Prof. em. Dr. Drs. h. c. Elmar Mittler, emittle@gwdg.de the overwhelming starting event for regaining the lost former Heidelbergian book treasures via microfilming, digitisation, and research. The development of the automation brought first the transparent library with worldwide connections. Nowadays, the libraries are in metamorphoses: on the one hand they deliver virtually their holdings boundless; on the other they provide information media of every kind of opportunities for the acquirement of knowledge via active and often collaborative design in addition. Supported by functional and often fascinating buildings libraries are becoming more and more user driven instead of librarian driven.

Keywords: Library planning; Bibliotheca Palatina; library architecture; library automation; user driven library

Inhalt

1 Opas Bibliothek ist tot . . . . . . . . . . . . . . 281

2 Ja mach nur einen Plan... Der Bibliotheksplan Baden-Württemberg. . . . . . . . . . . . . . . . 281

3 ... und mach dann noch 'nen zweiten Plan Bibliotheksplan 1973 und Bibliotheken '93. Wegbereiter eines goldenen Zeitalters der Bibliotheken in West und Ost . . . . . . . . . . . . . 282

4 Phönix aus der Asche. Die Ausstellung Bibliotheca Palatina in Heidelberg 1986 . . . . . . . . . . 285

5 Lasst den Leser an die Bücher-Bibliotheksbau in der Spätzeit der Gutenberggalaxis und darüber hinaus ................... 286

6 Die gläserne Bibliothek - von den lokalen Bibliothekssystemen zur Vernetzung in Verbundkatalogen und darüber hinaus. . . . . . . . . . . . . . 287

7 Von der Raupe zum Schmetterling - Die Metamorphose der Bibliotheken. . . . . . . . . . 288

8 Ein Wort zum Schluss . . . . . . . . . . . . . . 290

Bibliotheken sind Mittler zwischen Vergangenheit und $\mathrm{Zu}$ kunft. Sie dienen der adäquaten Sicherung vergangener (im weitesten Sinn) publizierter Medien mit dem Schwerpunkt schriftorientierter Materialien, ihrer Erschließung und ihrer Bereitstellung in Gegenwart und Zukunft. Dieser 
Transfer durch Bibliotheken hat sich in den vergangenen Jahrzehnten massiv gewandelt. Hier sollen einige Etappen der Transformation (im weiteren Sinne, nicht nur als digitale Transformation) angesprochen werden, an denen der Autor aktiv mitgewirkt hat.

\section{Opas Bibliothek ist tot}

Als ich 1967 die Freiburger Universitätsbibliothek von innen kennenlernte (und mich dabei an den Türen mit der Aufschrift „Kein Eingang“ orientierte, um meinen Weg im komplexen Gebäude zu finden) war ich beeindruckt und aufmüpfig zugleich. Beeindruckt von den konsequenten Methoden, Literatur in Katalogen zu erfassen und zugänglich zu machen, aufmüpfig gegen die Formen, in denen das immer wieder auch entgegen dem gesunden Menschenverstand geschah. Heute weiß ich, dass ich als Bibliotheksreferendar eine obsolet gewordene Version des Göttinger Bibliotheksmodells einer wissenschaftlichen Forschungsbibliothek kennengelernt habe. War es Heyne in Göttingen gelungen, durch die gezielte Auswahl der internationalen Literatur, die den Fortschritt der Wissenschaft dokumentierte, ihre sachliche Erschließung und nutzerfreundliche Bereitstellung die Bibliothek zum perfekten Instrument für die Forschung zu machen und sie in den wissenschaftlichen Diskurs $\mathrm{zu}$ integrieren, aber auch den Studenten dienstbar zu machen, so war diese Konzeption in Freiburg weitgehend pervertiert. Die Bibliothek lebte in splendid isolation. Natürlich kaufte sie nach dem Verständnis des jeweiligen Fachreferenten die hoch qualifizierte wissenschaftliche Forschungsliteratur für den Lehrkörper. Die Professoren aber nutzten die für ihre Forschungsgebiete relevante Literatur in den Institutsbibliotheken; dort fand sich auch die wichtigste Studienliteratur, deren Kauf in der Universitätsbibliothek (UB) weitgehend ausgeklammert blieb. Die Erwerbungen wurden systematisch aufgestellt, ohne dass die Benutzer direkten Zugang zu ihnen gehabt hätten - und das in das durchaus bewährte aber total veraltete Hartwig'sche System, bei dem Literatur zur Tschechoslowakei noch unter Österreich-Ungarn gesucht werden musste... Als Wolfgang Kehr während meiner Zeit am Bibliothekar-Lehrinstitut in Köln 1967 die Leitung der Bibliothek übernahm, hat er in Windeseile begonnen, die Bibliothek neu zu formieren. Die systematische Aufstellung wurde durch die Numerus-Currens-Aufstellung in vier Großgruppen ersetzt, neue Kontakte zu den Fachbereichen geknüpft und das Angebot für die Studierenden spürbar verbessert. Es kamen ihm einige Zeittrends entgegen. Das Gutachten des Wissenschaftsrats Wissen- schaftliche Bibliotheken ${ }^{1}$ zu Personal- und Mittelausstattung aus dem Jahr 1964 zeigte erste Auswirkungen und die Volkswagenstiftung hatte 1965 mit ihrem von Clemens Köttelwesch initiierten Förderprogramm zum Aufbau von Lehrbuchsammlungen vielgebrauchter Studienliteratur begonnen. Damit war der Aufbau von Freihandbeständen für die Studierenden möglich, von denen in der Folge die Attraktivität der Universitätsbibliothek neu entdeckt wurde - mit dem damit einhergehenden beeindruckenden Anstieg der Ausleihzahlen. Als ich aus Köln zurückkam, war schnell deutlich, dass Kehr und ich praktisch identische Vorstellungen von dem hatten, was an Veränderung in der Bibliothek geschehen sollte, und half ihm nach Kräften, dies umzusetzen. So konnte ich schon auf dem Bibliothekartag in Augsburg 1970 in Abwandlungen einer populären studentischen Wendung kess behaupten:

„Die dauernd steigenden Studentenzahlen haben auch das Gesicht der Universitätsbibliotheken einschneidend geprägt: Wir haben tief gestaffelte Bestände in den Lehrbuchsammlungen; wir bieten in den Lesesälen möglichst große Präsenzbestände und viele Semesterapparate an; wir haben vor, Teile der Magazine für Selbstbedienung durch den Benutzer zu öffnen; - kurz, vergleicht man die benutzerintensiven Bibliotheken von heute mit Universitätsbibliotheken der Vergangenheit, drängt sich einem der Satz auf: ,Opas Bibliothek ist tot“.“2

\section{Ja mach nur einen Plan... Der Bibliotheksplan Baden-Württemberg}

Bert Brechts Zeilen aus der Ballade von der Unzulänglichkeit menschlichen Planens (Dreigroschenoper) „Ja, mach nur einen Plan!/ Sei nur ein großes Licht!/ Und mach dann noch'nen zweiten Plan/ Gehen" tun sie beide nicht wurden ein scherzhaft oft verwendetes Zitat in der Arbeitsgruppe Bibliotheksplan Baden-Württemberg (ABBW) mit Ver-

\footnotetext{
1 Wissenschaftsrat (1964).

2 Mittler (1970) 288. Die Veröffentlichung dieses Artikels in ZfBB dies sei im Vorübergehen angemerkt - war für mich Anlass, über die Gründung einer anderen Bibliothekszeitschrift nachzudenken. Man weigerte sich, eine einfache Skizze abzudrucken, und verwies darauf, diese bei mir anzufordern. Mit Paul Kaegbein und Joachim Kuhlmann als Mitherausgebern und Klaus G. Saur als Verleger war es 1977 soweit, dass das erste Heft von BIBLIOTHEK - Forschung und Praxis erscheinen konnte. Format und Gestaltung der Zeitschrift wurden dann zum Vorbild für alle späteren im deutschsprachigen Raum gegründeten bibliothekarischen Zeitschriften wie ABI-Technik oder bit-online; schließlich stellte sich auch ZfBB darauf um.
} 
tretern von Universitäten und Bibliotheken, die Wissenschaftsminister Hahn 1968 berufen hatte; der Verfasser wirkte als ihr Sekretär. Anders als in Nordrhein-Westfalen lag der Akzent der Landesplanung nicht auf der Gründung neuer Hochschulen und der Organisation ihres Bibliothekswesens, der dort neben neuen Universitäten in Bochum und Bielefeld zur Gründung von fünf Gesamthochschulen (1972) sowie der Fernuniversität Hagen (1974) führte, die vom ebenfalls neu geschaffenen Hochschulbibliothekszentrum Nordrhein-Westfalen (1973) EDV-gestützte Dienstleistungen erhielten. ${ }^{3}$ Baden-Württemberg beschränkte sich auf die Neugründung einer „Eliteuniversität" in Konstanz, die mit einem einschichtigen Bibliothekssystem mit Freihandbeständen in systematischer Aufstellung und EDV-Unterstützung zum Vorreiter neuer bibliothekarischer Konzeptionen im Lande wurde; man konzentrierte sich darüber hinaus auf den Ausbau der traditionsreichen Universitäten Freiburg, Heidelberg und Tübingen sowie der ebenfalls seit langem gut etablierten Technischen Hochschulen in Karlsruhe und Stuttgart sowie die Weiterentwicklung der Fachhochschulen. Kernfrage der baden-württembergischen Planungsgruppe war die Frage, wie die traditionelle zweigliedrige Bibliotheksstruktur mit dem unkoordinierten Nebeneinander von Universitäts- und Institutsbibliotheken weiterentwickelt werden sollte. Der mit modernen Methoden durchgeführte Planungsprozess wurde in enger Verbindung mit dem Bibliotheksreferat des Ministeriums vorangebracht. Das ermöglichte die Umsetzung vieler der 1973 und 1975 veröffentlichten Empfehlungen noch während ihres Entstehens. ${ }^{4}$ So konnten Gesamtkataloge der Universitäten, die durch Kopieren und Zusammenordnen der Institutskataloge entstanden, praktisch aus dem Stand begonnen werden. Zusätzliche Etatmittel wurden bereitgestellt, so dass den Universitäts- und den Landesbibliotheken teilweise mehr Gelder zugewiesen wurden, als sie beantragt hatten - ein Vorgehen des Ministeriums, das damals auch bei den Universitäten möglich war, weil deren Bibliotheken damals in Baden-Württemberg noch je eine eigene Titelgruppe im Haushalt der Hochschulen hatten. Die erfolgreiche Transformation zu einem gegliederten Bibliothekssystem mit zentralen und dezentralen Elementen gelang auch deshalb, weil die rechtlichen Voraussetzungen in eine Novelle des Hochschulgesetzes übernommen wurden, in dem der Bibliotheksdirektor zwar „nur“ die Fachaufsicht über das Bibliothekspersonal erhielt, ihm damit aber ausreichende Zuständigkeiten z. B. für die Einführung

3 Nordrhein-Westfalen (1974) $49 \mathrm{ff}$.

4 Arbeitsgruppe Bibliotheksplan (1973) und (1975). einheitlicher Katalogregelungen zur Verfügung standen. Die Arbeitsgruppe Bibliotheksplan konnte damit in der praktischen Umsetzung sehr viel weiter kommen, als die Deutsche Forschungsgemeinschaft mit ihren Empfehlungen für die Zusammenarbeit zwischen Hochschulbibliothek und Institutsbibliotheken, ${ }^{5}$ die aber für die Argumentation sehr nützlich waren. So konnten die dort (auf Anregung von Wolfgang Kehr, Mitglied und späterer Vorsitzender der ABBW) empfohlenen $10 \mathrm{DM}$ pro Student für die Lehrbuchsammlung in die baden-württembergische Planung „übernommen“ werden. Damit aber war ein mit den Studierendenzahlen wachsender Etatposten geschaffen, der mit der Bereitstellung viel gebrauchter Literatur und dem sich dadurch ergebenden schnellen Anstieg der Bibliotheksnutzung die Stellung der zentralen Bibliotheken in den Universitäten völlig veränderten - eine Transformation, die auf diese Weise ohne große bibliothekarische Binnendiskussion zwischen forschungsorientierten Traditionalisten und benutzerorientierten Neuerern durchgesetzt werden konnte.

\section{3 ... und mach dann noch 'nen zweiten Plan - Bibliotheksplan 1973 und Bibliotheken '93. Wegbereiter eines goldenen Zeitalters der Bibliotheken in West und Ost}

Dissens und Abgrenzung war das traditionelle Kennzeichen der Bibliothekars- und Bibliotheksverbände: 1922 emanzipierten sich von dem 1900 gegründeten Verein deutscher Bibliothekare an wissenschaftlichen Bibliotheken (VDB) der VDV, der Verband Deutscher Volksbibliothekare (1969 VBB), die ihrerseits einen heftigen Richtungsstreit zwischen Anhängern der Bildungsbibliothek (Walter Hofmann) und der Informationsbibliothek (Paul Ladewig) austrugen; noch 1948 entstand der VdDB, der Verein der Diplombibliothekare an wissenschaftlichen Bibliotheken), um dieser Berufsgruppe eine ausreichende Vertretung gegenüber dem VDB zu ermöglichen. Da war es geradezu revolutionär, dass sich 1973 Öffentliche und wissenschaftliche Bibliotheken und die übrigen bibliothekarischen Sparten wie die bibliothekarischen Fachhochschulen im Deutschen Bibliotheksverband zusammen-

5 DFG (1970). 
schlossen. Gemeinsam legten sie im Bibliotheksplan 1973 einen Strukturplan für das deutsche Bibliothekswesen vor, der zur „Magna Charta überlegter Aufgabenverteilung und Kooperation im deutschen Bibliothekswesen“ wurde. ${ }^{6}$ Er war geprägt von Pragmatismus und Kompromissbereitschaft und ermöglichte einen entscheidenden Durchbruch für das Bibliothekswesen in Deutschland. Zwar sind die Richtwerte und Planungsvorgaben wohl nirgendwo vollständig erfüllt worden. Aber wenn z. B. die Öffentlichen Bibliotheken zwischen 1966 und 1990 ihre Bestände mehr als vervierfachen und das Personal nahezu verdoppeln konnten, ${ }^{7}$ ist das auch auf die durch den Bibliotheksplan 1973 gestärkte Öffentlichkeitswirksamkeit bibliothekarischer Argumentationen und Zielvorstellungen zurückzuführen. Der Bibliotheksplan passte in die Konsensgesellschaft der damaligen Bundesrepublik Deutschland, die sich strukturell neu und nachhaltig organisieren wollte. Damals waren die Ohren für den Rat der Fachleute in den Wissenschaftsministerien von Bund und Ländern ebenso offen wie in vielen Städten und Gemeinden, wobei insbesondere über die KGSt (Kommunale Gemeinschaftsstelle für Verwaltungsvereinfachung) unter bibliothekarischer Beteiligung Empfehlungen entstanden, ${ }^{8}$ die ebenso in den Bibliotheksplan 1973 einflossen wie die der Länderplanungen, insbesondere auch des Bibliothekplans Baden-Württemberg. Im Ergebnis kann man geradezu von einem goldenen Zeitalter für Bibliotheken sprechen.

Eine einvernehmliche Argumentation aller Bibliotheksverbände - des Institutionenverbandes DBV wie der Personalverbände - war das Ziel der Gründung der BDB, Bundesvereinigung Deutscher Bibliotheksverbände (heute BID) im September 1989. Sie kam im rechten Moment, um im Prozess der Wende in enger Zusammenarbeit mit ostdeutschen Bibliothekaren die Vereinigung des deutschen Bibliothekswesens zu strukturieren. Seit Januar 1990 gab es gemeinsame Tagungen abwechselnd in Ost und West (zuerst in Heidelberg, dann in Rostock-Warnemünde). ${ }^{9}$ Sie bereiteten den Weg zur Vereinigung der Bibliotheksverbände im Februar 1991 in Göttingen. Die gemeinsam entwickelten Planungsvorstellungen konnten schon vor der Wiedervereinigung in die Arbeit der späteren BundLänder-Arbeitsgruppe Bibliothekswesen eingebracht werden. ${ }^{10}$ Ziel war die schnelle Transformation bei den wissenschaftlichen wie den Öffentlichen Bibliotheken und sie

\footnotetext{
6 Plassmann et al. (1999) 206.

7 Umlauf (2008).

8 Mittler (2017a) 609f.

9 Mittler (1990) 356.

10 Bund-Länder-Arbeitsgruppe (1991).
}

gelang. Die Universitätsbibliotheken waren Stiefkinder des Bibliothekswesens der DDR. Die wissenschaftliche Forschung war nach sowjetischem, letztlich französischem Vorbild in der Akademie konzentriert; die Universitäten spielten eine Nebenrolle. Das wirkte sich auch bei der Zuteilung von Devisen für ausländische Literatur aus; selbst bei der Verteilung der westlichen Bücher, die nach dem Ende der Buchmessen in Leipzig verblieben, hatten die Universitätsbibliotheken nur nachrangige Plätze. Als sich die Grenze öffnete, galt es, schnell zu handeln. Als Sprecher der BDB bat ich damals Michael Hirsch vom Bundesministerium für Bildung und Forschung ein Dreijahresprogramm von je 5 Mio. DM für den Aufbau von Lehrbuchsammlungen aufzulegen, um die Attraktivität der Universitäten in Ostdeutschland zu stützen; es gelang, 15 Mio. DM noch für das Jahr 1990 bewilligt zu erhalten. Die Literatur sollte nach der Vorstellung des Ministeriums in westdeutschen Bibliotheken für die (damals noch) DDRBibliotheken erworben werden. Es gelang, die direkte $\mathrm{Zu}$ teilung an die ostdeutschen Bibliotheken zu erreichen. Das Ergebnis war beeindruckend: Da die Gelder bis zum Ende des Jahres 1990 ausgegeben werden sollten (es gelang dann eine Ausnahmeregelung bis März 1991 zu erwirken), haben die ostdeutschen Bibliothekare auf der Grundlage westdeutscher Titellisten vielgebrauchter Literatur in wenigen Monaten mehr westliche Literatur erwerben können, als vorher in einem Jahrzehnt. Man musste teilweise Tag und Nacht arbeiten. So kann man sagen, dass in der zweiten Hälfte des Jahres 1989 die Lichter in den Universitätsbibliotheken angingen, während sie wegen des Wegbrechens ihrer Märkte in den Fabriken mehr und mehr erloschen. Die Bibliothekare in den neuen Ländern empfanden es aber auch als eine Anerkennung ihrer fachlichen Kompetenz, dass man ihnen die Bewältigung dieser schwierigen Aufgabe zutraute; es erfüllte sie mit Stolz, sie erfolgreich bewältigt zu haben. Eine Verstetigung gelang dadurch, dass die Empfehlung der Bund-Länder-Arbeitsgruppe, die Universitätsbibliotheken der neuen Länder wie Bibliotheken neuer Universitäten für einen Zeitraum von 12 Jahren mit Erstausstattungsmitteln zu versehen, vom Wissenschaftsrat aufgegriffen und mit leichter Reduzierung umgesetzt wurde. So ist es gelungen, die Universitätsbibliotheken in einem guten Jahrzehnt, in dem sie über mehr Mittel verfügten als die westdeutschen Einrichtungen, zu ebenbürtiger Leistungsfähigkeit zu führen. Sie wurde durch Neubauten (oder neubaugleichen Ausbau kriegsbeschädigter Gebäude wie in Leipzig) an praktisch allen Standorten zusätzlich gefördert.

Die Öffentlichen Bibliotheken der DDR wurden von Partei und Regierung auch zur Verbreitung ihrer weltanschaulichen und politischen Vorstellungen breit unter- 
stützt. Selbst in den kleinsten Orten gab es Bibliotheken eine Ausstattung, die natürlich mit der Finanzierungsstruktur der Bundesrepublik, in der die Gemeinden für die Bibliotheken aufkommen müssen, nicht haltbar war. Das Bibliothekensterben füllte die Schlagzeilen der Presse nach der Wiedervereinigung. Doch auch hier gelang es, in der Ausnahmesituation der Wiedervereinigung Bundesmittel für den Aufbau neuer Bestände zu erhalten, die durch persönliches Engagement von Bundeskanzler Kohl noch einige Zeit fortgesetzt wurden, als sie in anderen Sparten schon ausgelaufen waren. Dabei standen die Bibliothekare nach der Wende praktisch vor dem Nichts. Ihre Buchbestände waren von einem Tag zum andern so gut wie obsolet geworden: Die marxistisch-leninistische Literatur allemal, aber auch die Sachbücher von den Reiseführern bis hin zu Heimwerkerliteratur musste ersetzt werden; schließlich standen auch die vorher so viel gefragten Romane regimekritischer DDR-Autoren plötzlich unbenutzt in den Regalen (nach dem Verkauf der niedrigen Auflagen in den Buchhandlungen waren sie vorher „Renner“, weil sie praktisch nur noch in den Bibliotheken zugänglich waren, die sie im Rahmen der Kontingentierung frühzeitig erhalten hatten). Es ist eine der größten Leistungen der ostdeutschen Bibliothekare, dass sie die Transformation ihrer Bibliotheken sehr schnell bewältigt haben: Mit weniger Bibliotheken und sinkendem Personalstand haben sie die Ausleihzahlen nach nur zwei Jahren in einigen Bundesländern auf ein höheres Niveau gebracht als vorher im „Leseland“ DDR. Auch hier half bei der Strukturierung und zukunftsorientierten Weiterentwicklung des Bibliothekswesens der Bibliotheksplan 1973, der im Planungspapier Bibliotheken '93 auf den neusten Stand gebracht wurde. 1994 konnte dieses auf dem ersten gesamtdeutschen Bibliothekskongress 1993 verabschiedet werden, war aber schon vorher bei Ministerien und Verbänden intensiv kommuniziert worden. ${ }^{11}$ Dieser Leipziger Kongress war auch ein wichtiger Schritt hin zu einem gesamtdeutschen Wir-Gefühl der deutschen Bibliothekare. Eine Besonderheit war darüber hinaus, dass die Leipziger Buchmesse in die Pfingstwoche verlegt wurde, um die große Zahl der bibliothekarischen Kongressbesucher auch für die in ihrem Bestand gefährdete Buchmesse zu gewinnen - eine gemeinsame Aktivität, die auf Jahre zu einer vertrauensvollen Zusammenarbeit von BDB und Börsenverein geführt hat. Sie führte z.B. zu der schon von Birgit Dankert als Nachfolgerin des BDB-Sprechers Mittler unter-

11 Mittler (1993b), BDB (1993), BDB (1994) mit Vorwort von Mittler sowie Kasch (2008) $24 \mathrm{f}$. sieht den veralteten Bibliotheksplan 1973, der damals aber nachgedruckt wurde, als das in der damaligen Zeit vorbildliche Strukturpapier. schriebenen Bremer Erklärung zur gemeinsamen Herausforderung von Bibliotheken und Buchhandel durch die Online-Entwicklung. ${ }^{12}$ Mit dieser Vereinbarung suchte man in der damals noch offenen Entwicklungssituation die Kooperation zu stärken. Leider ist diese Aktivität in den sich schnell verhärtenden Fronten im Kampf um das Urheberrecht nicht zum Tragen gekommen. Daran waren die Bibliotheken nicht ganz unschuldig. Mit Unterstützung des BMBF hat die Technische Informationsbibliothek damals z.B. eine Werbekampagne gestartet, um über ihren gut organisierten Dokumentlieferdienst zusätzliche Einnahmen zu generieren. Von ausländischen Verlegern wurde mir immer wieder berichtet, dass durch den spektakulären Auftritt bei der London Book Fair insbesondere auch die amerikanischen Verleger auf die für sie unverständliche Regelung des deutschen Kopierrechts aufmerksam wurden, dass ohne explizite (in den USA übliche) Zustimmung Erstellung und Versand von Kopien für persönlichen Gebrauch bei Abgabe einer Kopiergebühr erlaubt (was in der Folge bis zu einer Demarche des amerikanischen Botschafters bei der deutschen Regierung führte). Dabei beruht die deutsche Regelung auf einer Schranke im internationalen Urheberrecht, die ein entsprechendes Vorgehen erlaubt, wenn dadurch die normale Verwertung durch die Rechteinhaber nicht unzumutbar eingeschränkt wird. Eine aggressive Vermarktung von Bibliotheksbeständen mit dem Ziel, Einnahmen durch Kopien zu erzielen, konnte aber durchaus die Anwendung der Schranke gefährden. Für elektronische Medien wurde später auf europäischer Ebene geregelt, dass Schranken in einem Lizenzvertrag ausgeschlossen werden können. Die Kopierkampagne der TIB war Teil der ganz auf die neuen Entwicklungen des Internet ausgerichteten Bibliothekspolitik Uwe Rosemanns; in den Anfangszeiten des Internet war die Vorstellung verbreitet, dass es zu einer Zentralisierung der Angebote bei wenigen Anbietern führen werde. Uwe Rosemann suchte deshalb, die TIB zu einem zentralen Dienstleister nicht nur für seine Nutzer, sondern auch für die übrigen Bibliotheken zu machen. Ganz offen sprach er immer wieder davon, dass er Mittel aus deren Etat „einsammeln“ wolle. Durch das Einrichten des Niedersachsenkonsortiums mit der Zentrale bei der SUB Göttingen konnte aber das Gegenteil erreicht werden: Mit einem relativ geringen zentralen $\mathrm{Zu}$ satzbetrag wurde mit großen Zeitschriftenverlagen vereinbart, dass alle beteiligten Bibliotheken kostenfrei auf die im Lande abonnierten elektronischen Zeitschriften zugreifen können - auch auf die der TIB. Der damalige Wissenschaftsminister Thomas Oppermann konnte für die Bereit-

12 Börsenverein (1996). 
stellung der notwendigen Mittel gewonnen werden (Abb. 1). Seiner sei hier besonders gedacht; er war dem Autor freundschaftlich verbunden. Auch als Bundestagsabgeordneter hat Oppermann sich immer wieder für die Bibliotheken eingesetzt, so beim Urheberrechts-Wissensgesellschafts-Gesetz (UrhWissG) 2017. Am 15.10.2020 ist er plötzlich verstorben; so konnte er am Symposium Transfer und Transformation nicht mehr teilnehmen, bei dem er ein Grußwort sprechen wollte.

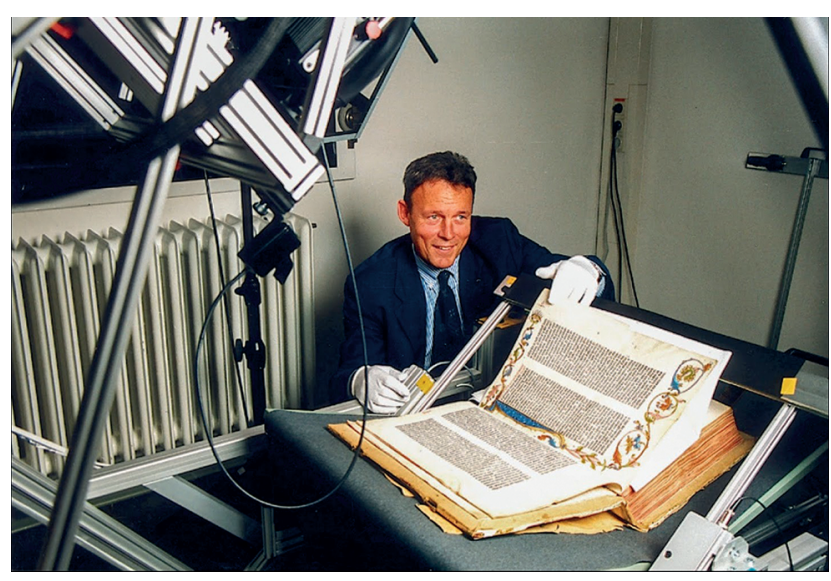

Abb. 1: 1999: Wissenschaftsminister Thomas Oppermann bei einem Probescan der Digitalisierung der Göttinger Gutenbergbibel

\section{Phönix aus der Asche. Die Ausstellung Bibliotheca Palatina in Heidelberg 1986}

Inzwischen hatte in Westdeutschland und weit darüber hinaus ein anderes Ereignis fünf Monate lang eine historische Bibliothek in den Mittelpunkt öffentlichen Interesses gerückt: Die Bibliotheca Palatina, Heidelbergs wertvollste Bücherbestände, die sich bei Ankunft des päpstlichen Abgesandten Leone Alacci Ende November 1622 noch getrennt hauptsächlich auf den Emporen der Heiliggeistkirche, aber auch auf dem Schloss und in der Universitätsbibliothek befanden. Der Lektor für Griechisch hat 1623 alles, was er für wertvoll hielt, in 192 Kisten (von denen 12 für ihn bestimmt waren) in einem mehrmonatigen Transport nach Rom gebracht, womit - wie Papst Gregor XV. es in seinem Dankschreiben an Maximilian I. von Bayern ausdrückte die Waffen der Ketzer zu Schutzschilden der rechten Konfession werden sollten. Es war eine komplexe diplomatische Aufgabe, zum 600. Jubiläum der Gründung der Universität Heidelberg die Bibliothek in repräsentativer Auswahl nach Heidelberg für eine Ausstellung zurückzuholen. Lange Zeit blieb unklar, ob eine Ausleihe geneh- migt würde; die Zusage, gut 600 erbetene Handschriften und Drucke wirklich nach Heidelberg transportieren zu können, kam erst sehr spät. Der Präfekt Alfons M. Stickler, der im Verlauf der Verhandlungen bis zum Kardinalbibliothekar aufstieg, und sein Nachfolger als Präfekt, Leonard Boyle, haben es schließlich möglich gemacht, dass sich monatelang Schlangen von Besuchern bildeten, um Palatina Superstar kennenzulernen, wie die Heidelberger RheinNeckar-Zeitung noch kürzlich in einem Rückblick auf die Ausstellung titelte. ${ }^{13}$ Für knapp 4 Monate war sie geplant, wegen des großen Erfolges durfte sie um einen Monat verlängert werden. Mit überwältigenden 272000 Besuchern wurde wohl eine für Bibliotheksausstellungen nie wieder erreichbare Rekordmarke gesetzt. In der mehrjährigen Vorbereitungszeit konnte ich in einer Reihe von einwöchigen Aufenthalten den gesamten bekannten vatikanischen $\mathrm{Pa}$ latinabestand auf seine Ausstellbarkeit und die Eignung der Druckschriften zur Verfilmung für die geplante Mikroficheausgabe durchsehen; an der wissenschaftlichen Auswahl und Beschreibung der Ausstellungsstücke war ein Team beteiligt, das mit der Zeit auf 24 Personen anwuchs. Dabei hatte eine Heidelberger Rundfunkjournalistin, der ich im Vorfeld der Ausstellung nicht ohne Stolz die im unterirdischen Magazin für den Transport zusammengestellten Bände zeigte, spontan reagiert mit: „Damit wollen Sie die Heidelberger Bürger begeistern?" Erst als ich ihr an ein paar Beispielen die Schönheit und die Bedeutung der Bücher für ihre Besitzer erläutert hatte, war sie überzeugt, dass sich auch viele andere dafür interessieren könnten. Die Ausstellung hat in exemplarischer Weise gezeigt, wie aufschlussreich, ja aufregend es sein kann, Bibliothek als Geschichte zu präsentieren, sie als ein Zeugnis der Interessen, der Weltanschauungen aber auch der Kämpfe und Niederlagen ihrer Besitzer sichtbar zu machen - für die Heidelberger, aber auch (wie man dort gern sagt) den Rest der Welt. Für mich selbst war es ein Erlebnis, wie die faszinierende Welt der Palatina wieder in wenige Regalmeter scheinbar unattraktiver Buchrücken zusammenfiel, als ich sie im Dezember 1986 in den Vatikan zurückgebracht hatte. Mit der Mikroficheedition der Drucke, für die mehr als 1600 vorher unbekannte Werke ermittelt werden konnten, ${ }^{14}$ und der Digitalisierung der Handschriften ${ }^{15}$ ist sie aber inzwischen eine Bibliothek, die virtuell nicht nur für Heidelberg zurückgewonnen werden

13 Neumayr (2021).

14 Boyle und Mittler (1989-1995), Mittler (1999).

15 Probst (2018), Adalbert Roth, der ehemalige Leiter der Druckschriftenabteilung der Biblioteca Vaticana gebührt das Verdienst, den Weg für die vertragliche Abmachung zur Digitalisierung auf meine Bitte geöffnet zu haben. Ihm sei herzlich dafür gedankt. 
konnte, sondern für die ganze Welt geöffnet worden ist. ${ }^{16}$ Die vielen Forschungsarbeiten, die nicht zuletzt angeregt durch die Ausstellung, inzwischen erschienen sind, tragen mit dazu bei, diesen einst scheinbar verlorenen „Schatz, der nicht zu schätzen“, zu heben.

\section{Lasst den Leser an die Bücher - Bibliotheksbau in der Spätzeit der Gutenberggalaxis und darüber hinaus}

Waren die Bibliotheken nach dem Zweiten Weltkrieg zunächst noch ganz am Ideal der dreigeteilten Bibliothek orientiert, bei der man schon stolz darauf war, wenn Leihstelle, Lesesaal und das geschlossene Magazin optimal um den Zettelkatalog herum gruppiert wurden, wie das z.B. beim Neubau der Universitätsbibliothek Mainz gelungen ist, so sah das 1983 ganz anders aus: Mit dem Motto Lasst den Leser an die Bücher wurde in der Heidelberger Universitätszeitschrift Ruperto-Carola die Neukonzeption der bibliothekarischen Services im Rahmen der Sanierung des 190. eröffneten Gebäudes der Universitätsbibliothek Heidelberg vorgestellt. ${ }^{17}$ Wechselnde Planungskonzeptionen der Universität hatten dazu geführt, dass ein geplanter Neubau im Neuenheimer Feld nicht realisiert wurde, weil die Universität sich dazu entschloss, die Geisteswissenschaften in der Innenstadt zu belassen. Das dort schon Anfang des 20. Jahrhunderts für eine Erweiterung der Magazine vorgesehene Gelände aber war für das sogenannte Triplexgebäude (Mensa, Seminarräume, Tiefgarage) verbaut worden, in dem der Bibliothek nur marginale Flächen zugewiesen wurden. So blieb die UB Heidelberg als eine der wenigen großen Bibliotheken Deutschlands ohne neues Gebäude. Bei der Sanierung musste versucht werden, die zeitgerechten Funktionen zu ermöglichen, die bei Neubauten selbstverständlich erfüllt wurden: mehr Leseplätze für die Nutzer, freier Zugang zu möglichst vielen Beständen und ausreichende Aufstellungsmöglichkeiten für die schnell wachsende Zahl der Bücher - sowie nicht zuletzt auch eine adäquate Unterbringung des Personals. Das alte Heidelberger Bibliotheksgebäude, von dem Jakob Wille, der erste darin amtierende Direktor, festgestellt hat, es gelte in Fachkreisen allgemein als ein Beispiel dafür, wie

16 Mittler (2007).

17 Mittler (1983), der Sanierungsprozess ist dokumentiert in Mittler (1989). man Bibliotheken nicht bauen sollte, ${ }^{18}$ erwies sich als erstaunlich flexibel. Durch Verdichtung und zusätzlichen Ausbau konnten das Personal und die hybride Kombination der damaligen Informationsmittel (Band-, Zettel-, Mikrofiche- und EDV Kataloge; gedruckte und digitale Bibliographien) im „Schlossteil“19 untergebracht werden. ${ }^{20}$ Zwei Geschosse im Magazinteil wurden mit den Neuzugängen der letzten Jahrzehnte (allerdings in Numerus-CurrensAufstellung) bestückt; die beiden darüber liegenden ehemaligen Magazingeschosse wurden zu Lesebereichen umfunktioniert. Es blieb aber noch ein scheinbar unlösbares Problem: Große Teile der geisteswissenschaftlichen Altbestände waren in eine angemietete ehemalige Zigarrenfabrik ausgelagert. Wohin damit in der eng bebauten Heidelberger Innenstadt? Es fand sich eine akzeptable Lösung: die allerdings nur unter großen Mühen realisierbare unterirdische Anbindung eines Tiefmagazins im Innenhof der Neuen Universität. Damit aber waren die Grundlagen für eine schon während des Bauvollzugs erkennbar große Nutzerakzeptanz und den (Wieder-)Aufstieg der Heidelberger Bibliothek zu einer der leistungsfähigsten deutschen Bibliotheken gelegt. Bei der Heidelberger (Not-)Lösung wurde ganz bewusst auf den Einsatz der Klimatisierung verzichtet. Sie war Kennzeichen der damals gebauten „Bibliotheksmaschinen“, wie sie etwa in Freiburg gebaut wurde. Die rund 22000 qm gegenüber ca. 2400 qm Nutzfläche des Neorenaissancebaus für ein Gymnasium, das auf dem gleichen Gelände stand, bedeuteten fast eine Verzehnfachung - zum Preis einer Vollklimatisierung, die dem Gebäude den Ruf einer Energievernichtungsmaschine einbrachte. Das Universitätsbauamt war deshalb froh, den asbestverseuchten oberirdischen Teil abreißen und durch einen klimasparenden Neubau ersetzen zu können. Dabei verschwand auch die von der Bevölkerung nie akzeptierte Betonfassade aus der Zeit des Brutalismus - wurde allerdings durch eine hochmoderne, man möchte fast sagen modische, Glasfassade ersetzt, die wegen ihrer Blendwirkung in manchen Jahreszeiten zum Schrecken der Autofahrer geworden ist. Schon im ersten Freiburger Neubau wurde aber die Konzeption großer, von den Benutzern gut angenommener Lesebereiche, Frei-

18 Wille (1911).

19 Der Architekt Josef Durm hatte den südlichen Teil des Gebäudes mit einer prächtigen Fassade versehen die - wie ich inzwischen ermitteln konnte - Strukturen des Schlossbaus von Gaillon an der Seine aufgenommen hat.

20 Die Sanierung ist in Mittler (1989) dokumentiert. Die Verdichtung konnte inzwischen durch Herauslösen der bewusst wieder entfernbar gestalteten metallenen Teilzwischengeschosse zurückgebaut werden, vgl. Nissen (2020). 
handbestände der neueren Literatur mit Numerus-Currens-Aufstellung und Unterbringung der Altbestände in unterirdischen Magazinen realisiert, wie sie auch in Heidelberg in bescheideneren Dimensionen zu finden ist. Bei den neuen Universitäten dagegen wurde (zunächst) der gesamte Bestand systematisch geordnet frei zugänglich gemacht - eine Aufstellungsform, die besonders viel Platz benötigt und sich auch hier wegen des hohen Energieaufwandes als nicht nachhaltig erwies. Diese (oft in universitäre Gesamtanlagen integrierten) Gebäude waren die deutsche Version der „fully flexible library“, wie sie vor allem in England im Anschluss an Metcalf und Ellsworth vom britischen Architekten Faulkner-Brown propagiert und gebaut wurde. ${ }^{21}$ Demgegenüber wurde das Konzept der postmodernen Bibliothek im Rahmen der Seminare der LIBER architecture group ${ }^{22}$ entwickelt und bei Bibliothekaren wie bei Architekten propagiert. Dabei sollten Funktionalität und Technik mit Ästhetik und symbolischer Ausstrahlung verbunden werden. ${ }^{23}$ Das neue Gebäude der Niedersächsischen Staats- und Universitätsbibliothek kann dafür als gelungenes Beispiel gelten. ${ }^{24}$ Um große Baumaßnahmen möglich zu machen, war es in der Regel erforderlich, intensive Öffentlichkeitsarbeit zu realisieren - eine Aufgabe, die für Bibliothekare neu war. Wolfgang Kehr hat das in Freiburg vorbildlich begonnen; der Autor hat es in Karlsruhe und Heidelberg erfolgreich fortsetzen können. ${ }^{25}$ Lag noch im Neubau der 1993 eröffneten SUB Göttingen das Augenmerk auf den Magazinen und den mit umfangreichen Buchbeständen ausgestatteten Leselandschaften, so rückte bald die Konzeption der Learning Library mit der Kombination von Büchern, Rechnern und multimedialen Angeboten bis hin zur Produktionsunterstützung, Beratungsangeboten sowie der Bereitstellung von Gruppenarbeitsräumen im 7/24-Betrieb (24 Stunden Öffnungszeit an 7 Tagen in der Woche) in den Mittelpunkt des Interesses. In dessen Fortentwicklung werden heute die Benutzeraktivitäten bewusst in die kollaborative Bibliothek integriert, die auch den aktiven Wissenserwerb mit nicht-textuellen Zeichensystemen z.B. im Makerspace der SLUB in Dresden ermöglicht. ${ }^{26}$ Vor allem bei den Öffentlichen Bibliotheken sind dabei spektakuläre Neubauten entstanden, von denen als jüngeres Beispiel das Oodi

21 Metcalf et al. (1971).

22 Sie wurde nach der Heidelberger Tagung 1980 vom Autor begründet, vgl. Neue Probleme (1981), https://libereurope.eu/working-grou p/liber-architecture-working-group-lag/.

23 Bisbrouck und Mittler (1997), McDonald (2006).

24 Mittler (2003).

25 Mittler (2004).

26 Bonte (2017). in Helsinki genannt sei mit seiner Kombination vom Buchbestand über die Videoangebote bis zum 3D-Druck, und das in einer architektonisch faszinierenden Umgebung, die auch die Natur einbindet.

\section{Die gläserne Bibliothek - von den lokalen Bibliothekssystemen zur Vernetzung in Verbundkatalogen und darüber hinaus}

Die Durchschaubarkeit der zweischichtigen Bibliotheksorganisation der alten Universitäten suchte man in den 70er- und 80er-Jahren noch mit Kopie und Zusammenordnen der Katalogkarten zu erreichen. ${ }^{27}$ Wenn man bedenkt, dass die Kapazität der für das in Baden-Württemberg bei der Ausleihverbuchung eingesetzte System OLAF gerade einmal dafür reichte, die Signaturen der ausgeliehenen Bände zu verwalten, wird verständlich, dass die elektronische Datenverarbeitung damals noch nicht auf einem Stand war, der den Ansprüchen der Bibliotheken genügen konnte. Doch das rasante Tempo der Entwicklung ermöglichte es dem HBZ Nordrhein-Westfalen schon 1973 die fünf neuen Gesamthochschulen mit Mikrofichekatalogen auszustatten. Das damals noch sehr ehrgeizig erscheinende, inzwischen längst überall realisierte Ziel der Baden-Württembergischen Bibliotheksplanung war es demgegenüber, einen Verbund zu schaffen, in dem die Katalogdaten gemeinsam erfasst werden und als Verbundkatalog zugänglich sein sollten, lokal aber Rechner für die aus dem Verbund gespeisten Online-Kataloge und die Daten der Ausleihe zur Verfügung stehen. ${ }^{28}$ Diese Konzeption konnte ansatzweise zum ersten Mal mit dem System HEIDI an der Universitätsbibliothek Heidelberg verwirklicht werden. Dort wurde das mit IBM entwickelte Dortmunder DOBIS eingesetzt, das zunächst noch im Offline-Betrieb Daten von der Verbundzentrale des Südwestverbundes in Konstanz einspielen konnte. ${ }^{29}$ Der für das lokale System eingesetzte IBM-Rechner wurde im Rechenzentrum der Universität aufgestellt und technisch betreut; mit dem RZRechner gekoppelt ermöglichte er über das lokale IBMNetz in den angeschlossenen Instituten nicht nur den $\mathrm{Zu}$ griff auf den UB-Katalog und die Ausleihdaten, sondern auch schon die EDV-Katalogisierung einiger Instituts-

27 Dorsch (1985).

28 Arbeitsgruppe Bibliotheksplan (1975) $245 \mathrm{ff}$.

29 Mittler und Dörflinger (1988) und Mittler (1989). 
bibliotheken. Ein erster Schritt zur „gläsernen“ Bibliothek war getan. Doch dabei kam Widerstand von unerwarteter Seite, den man sich heute kaum noch vorstellen kann. Die damals in Deutschland verbreitete geradezu feindliche Einstellung zum Computer führte zu Widerstand bei Personalrat und Personal. ${ }^{30}$ Die damals in Heidelberg geführte intensive innerbibliothekarische Diskussion kann man als interessantes Zeitdokument in Beiträgen der Theke, dem Informationsblatt der Mitarbeiter im Bibliothekssystem der Universität Heidelberg, nachlesen. ${ }^{31}$ Es ergab sich in dieser Zeit eine Art unheiliger Allianz zwischen Gewerkschaften und Ministerien. Schürten die einen die Angst vor dem Verlust von Arbeitsplätzen verlangten die anderen, dass man die Einsparmöglichkeiten durch Rationalisierung mit Hilfe der EDV beziffere. Mit dem Argument, dass nur bei EDV-Einsatz das außerordentliche Wachstum der Ausleihen und Neuerwerbungen bewältigt werden könne, ließen sich beide Seiten zufrieden stellen; dabei kam bei der UB Heidelberg noch der Hinweis auf den gegenüber vergleichbaren Bibliotheken des Landes unterdurchschnittliche Personalbestand hinzu.

In den 1990er-Jahren wurden die Bibliotheken zunehmend zu Vorreitern der EDV-Entwicklung in Deutschland. Das im Bibliotheksplan Baden-Württemberg konzipierte Verbundsystem mit zentraler Datenführung und lokaler Datenhaltung ließ sich in Deutschland erstmals 1991 durch die Übernahme des PICA-Systems aus den Niederlanden verwirklichen - eine Entscheidung des Niedersächsischen Bibliotheksverbundes in Abstimmung mit der Deutschen Bibliothek, die über Europa hinaus teilweise ungläubiges Erstaunen erregte, aber letztlich dazu führte, dass Deutschland sich globalen Netzen wie dem WorldCat einbinden konnte. ${ }^{32}$ Durch die EDV-Unterstützung auch der Fernleihe konnte das Ziel der gläsernen Bibliothek auf den ganzen Verbund ausgedehnt werden. Doch es geschah weit mehr: Mit dem Digitalisierungsprogramm der DFG und der Einrichtung von Digitalisierungszentren in Göttingen und München gelang es, Anschluss an die internationale Entwicklung zu finden; mit der Einführung von Internetangeboten übernahmen die Öffentlichen Bibliotheken eine führende Rolle bei der Verbreitung innovativer Dienste. Mit dem Konzept der Kombination digitaler Ausgaben mit einer gedruckten (print-on-demand-)Ausgabe war man beim 2002 gegründeten Göttinger Universitätsverlag den meisten deutschen Wissenschaftsverlagen um mehr als ein Jahrzehnt voraus (wobei die digitalen Aus-

30 Vgl. dazu Cronin (1983).

31 Dietrich (1984), Türk (1984), Mittler (1985), Türk und Brose (1985), Mittler (1986b), Riemschneider et al. (1986).

32 Mittler (2001). gaben beim Universitätsverlag in der Regel open access bereitgestellt werden). $\mathrm{Zu}$ einer Zeit, in der die Innovationskraft und -willigkeit in Deutschland zu erlahmen schien, waren die Bibliotheken treibende Kräfte. Konzeptionell hatten sie die Entwicklung klar im Blick, wie z. B. das erstmals auf dem Bibliothekartag 1995 in Göttingen vorgelegte BDB-Papier Bibliotheken im Zeitalter der Datenautobahnen und internationalen Netze zeigt, das auch in die repräsentative Sammlung Deutschlands Weg in die Zukunftsgesellschaft aufgenommen wurde. ${ }^{33}$ Soweit die Bibliotheken die Zukunftstrends erkannten und sich schnell auf sie umstellten, konnten sie eine aktive Rolle bei der Transformation der Forschung und der Universitäten spielen. Dazu verhalfen die wachsenden Möglichkeiten auf Länder- und Bundesebene, aber auch bei der Europäischen Union, Fördermittel für zukunftsorientierte Projekte zu erhalten. In der SUB-Göttingen wurde eine Forschungsund Entwicklungsabteilung geschaffen, um die Projektarbeit zu professionalisieren und den Transfer in die Praxis zu verbessern. ${ }^{34}$

\section{Von der Raupe zum Schmetterling - Die Metamorphose der Bibliotheken}

Doch in der breiten Öffentlichkeit wurden die zukunftsträchtigen Entwicklungen in den Öffentlichen und wissenschaftlichen Bibliotheken kaum wahrgenommen. Vom Tod der Bibliotheken zu schreiben, war eine Zeitlang in der Presse en vogue. Noch 2013 textete Kathrin Passig in der ZEIT, als habe sie noch nie eine moderne Bibliothek benutzt: „Bibliotheken sind Papiermuseen. Wenn sie sich nicht bald grundsätzlich wandeln, haben sie kaum noch eine Existenzberechtigung. Was sie können, kann das Internet besser. “35 Und selbst Bibliothekare pflichteten bei, wenn Rafael Ball 2016 in einem Interview dazu aufforderte „Bibliotheken: Weg damit!“, weil alles schon im Internet vorhanden sei. ${ }^{36}$

Dabei erwerben die Bibliotheken nicht nur in großem Umfang digitale Bestände und stellen sie ihren Nutzern bereit. Mit ihren digitalen Angeboten haben sie längst den „Kokon“ ihrer Mauern hinter sich gelassen. Die spektakuläre Digitalisierung der Göttinger Gutenbergbibel 2000,37

33 BDB (1996)

34 Mittler (2013).

35 Passig (2013).

36 Furger (2016).

37 http://gutenbergdigital.de. 
mit dem der Mann des 2. Jahrtausends sozusagen auch zu einem Mann des 3. Jahrtausends gemacht werden konnte, ist ein gutes Beispiel dafür. ${ }^{38}$ Neben großen Projekten der Massendigitalisierung arbeitet man erfolgreich daran, weltweit verstreute historische Bibliotheken virtuell zu rekonstruieren, wie die Beispiele des Virtuellen Skriptorium St. Matthias in Trier ${ }^{39}$ oder der Bibliotheca Laureshamensis digital $^{40}$ zeigen.

Auf dem Weg zum allgegenwärtigen, die gesamte Lebenswelt durchdringenden Internet, das vollständig in unser alltägliches Handeln integriert ist, bieten die Bibliotheken nicht nur Zugang zum Internet, sondern sie sind selbst Knoten des Internet. Aber die Bibliotheken haben auch erkannt, dass e-only nicht der Königsweg ist, sondern man dem Benutzer die Wahl bieten muss, nach Bedarf zwischen virtuellen und örtlich gebundenen bzw. digitalen und analogen Angeboten zu wechseln.

Fluide Medien brauchen stabile Institutionen. Allein die Bibliotheken sind in der Lage, in internationaler Vernetzung die verlässliche globale Infrastruktur für Information und Wissenschaft zu bilden, indem sie

- kooperativ die umfassende Zugriffssicherung auf informations- und forschungsrelevante digitale Daten und Dokumente garantieren,

- genuine lokale Sammlungen und Forschungsdaten international zugänglich machen (was sie mit ihren Repositorien tendenziell schon realisieren),

- in den einzelnen Ländern arbeitsteilig in nationaler Verantwortung und/oder fachlicher Differenzierung außerhalb der Bibliotheken liegende Daten, insbesondere Verlagsangebote, einbeziehen.

Dabei ist die dauerhafte Verfügungsgewalt über die Informationsressourcen die entscheidende Voraussetzung für Dienste, die interaktives, anreicherndes und veränderndes Arbeiten mit den bereitgehaltenen Inhalten erlauben. ${ }^{41}$ Nur so kann man sie in das entstehende Ecosystem kontextualisierter und vernetzter Datenbestände integrieren. Dabei darf man sich nicht mehr nur auf die nachträgliche Erschließung - sprich Semantisierung - konzentrieren. Eine neue Kultur des semantischen Publizierens ist erfor-

38 Wie wenig die Öffentlichkeit mit derartigen bibliothekarischen Aktivitäten rechnete, mag man daran sehen, dass ein Foto, das Martin Liebetruth am Scannertisch bei der Digitalisierung der Gutenbergbibel zeigt, im Spiegel mit der Bildunterschrift veröffentlicht wurde: „Alte Folianten versöhnen den Bibliothekar mit seinem schweren Los“, vgl. Köster (2001).

39 http://www.stmatthias.uni-trier.de/.

40 https://www.kloster-lorsch.de/digital/digitale-bibliothek.

41 Ceynowa (2015) 275. derlich. Diese Publikationen sind über Links zur Literatur hinaus mit Forschungs-, Geo-, Statistik- und vielfältigen anderen mit den Texten verbundenen Daten bis hin zu Programmen zur weiteren Bearbeitung verknüpft, auf die ebenfalls ein direkter Zugriff erfolgen kann. ${ }^{42}$ Mit Recht kümmern sich deshalb Bibliotheken um wissenschaftliche Schreib- und Editionsprozesse, damit „die Potenziale der Anreicherung, Interoperabilität, Verarbeitbarkeit und Verlinkung von Inhalten und Objekten ausgeschöpft werden“. ${ }^{43}$ Open Science ist die Zielrichtung der Entwicklung, für die Bibliotheken nicht nur die Open-Access-Publikation fördern müssen. Es muss alles getan werden, damit jeder Wissenschaftler, aber auch den Studierenden, die in Zukunft weitgehend digital studieren, forschen und publizieren wollen, eine sichere Infrastruktur zur Verfügung steht, die ihnen professionelle Unterstützung von der Erfassung der Daten und ihrer Bearbeitung über die Publikation bis hin zur Langzeitarchivierung bietet. Es ist der Weg „von der Literaturversorgung zum Rundum-sorglos-Service für Forschung und Studium“, ${ }^{44}$ den die Bibliotheken beginnen, gangbar zu machen. Er wird z. B. im Strategiepapier der Sächsischen Landesbibliothek SLUB 2025 detailliert konzipiert und in Abteilungen wie dem SLUB Text$L a b$ und dem SLUB Open Science Lab konkret umgesetzt. ${ }^{45}$ Die umfassende Digitalisierung aller Lebensbereiche ermöglicht es auch, den „kollaborativen Wissensaufbau mit nicht-textuellen Zeichensystemen im SLUB Makerspace“ anzubieten. ${ }^{46}$ So entstehen neue Freiräume für die Nutzer der Bibliothek, die nicht mehr nur als Wissensspeicher, sondern als „Werkstatt der Wissenschaft“ ${ }^{47}$ wirken. Am deutlichsten hat es Joyce Valenza auf den Punkt gebracht, wenn sie fordert, die Bibliothek müsse sozusagen vom Lebensmittelladen zur Küche werden. ${ }^{48}$ Die Öffnung der Bibliotheken hat in Öffentlichen Bibliotheken in Dänemark sogar dazu geführt, dass Bibliotheken auch in der Zeit ohne Bibliothekare den Nutzern als Treffpunkt, z.B. für Diskussionsforen, zugänglich gemacht werden. ${ }^{49}$ Weit weg sind die Zeiten, in denen Leser von der einschüchternden Aufsicht an einer zentralen Theke in strahlenförmig angeordneten Leseplätzen nach der Konzeption des für Gefängnisbauten entworfenen Panoptikum überwacht

42 Der bekannteste Prototyp wurde schon 2009 veröffentlicht: Shotton et al. (2009). Vgl dazu auch Mittler (2012) $61 \mathrm{ff}$.

43 Degkwitz (2016) 29.

44 Mittler (2017b) 219.

45 Bonte und Muschalek (2019).

46 Bonte (2020) 381, Bonte (2017).

47 Horstmann (2014).

48 Valenza (2008).

49 Schulz (2015) 210. 
wurden, wie im Lesesaal der Bibliothek des British Museum und vielen anderen Bibliotheken der Welt. ${ }^{50}$ Der Benutzer wird zunehmend zum Partner, der zusätzliche Initiativen und Kenntnisse in die Bibliothek bringt. ${ }^{51}$ Wir sind auf dem besten Weg von der „Bibliothekarsbibliothek“ hin zur „Benutzerbibliothek“. Vielleicht können mutige Bibliothekare der Zukunft damit die Bibliothek realisieren, von der ich auch als Bibliothekar immer geträumt habe.

\section{Ein Wort zum Schluss}

Wie beim Symposium Transfer und Transformation seien mir abschließend ein paar persönliche Worte erlaubt.

Es gibt Einiges, von dem ich glaube, sagen zu können, dass es ohne mich wahrscheinlich nicht zustande gekommen wäre. In Karlsruhe konnte ich die Grundlagen des Neubaus der Badischen Landesbibliothek schaffen, in Heidelberg das Gebäude von 1906 revitalisieren und durch ein Tiefmagazin erweitern sowie die Ausstellung Bibliotheca Palatina diplomatisch vorbereiten und inhaltlich konzipieren. In Göttingen waren die Sanierung des Altbaus mit der Paulinerkirche und die Idee, die Buchbestände günstig gelegener Institute in den ehemaligen Klinikbauten mit einem Neubau $\mathrm{zu}$ einem bibliothekarischen Kontinuum zusammenzuschließen, das Kern des Kulturwissenschaftlichen Zentrums bildet, herausragende Projekte; auch die Einführung von PICA und der Aufbau des Gemeinsamen Bibliotheksverbunds gehören dazu. Dies alles habe ich in der Regel mit anderen zusammen getan, und Vieles von dem, was ich angestoßen habe, ist später durch andere gestaltet und zum Erfolg geführt worden. Es gibt aber auch Vieles, was ich nicht erreichen konnte. Wie bei Anträgen war für mich eine Erfolgsrate von 30 \% meistens schon ein guter Wert.

Es gibt Viele, die mich gefördert haben. Ich will nur drei herausgreifen und bitte dafür alle, die ich nennen möchte, aber hier nicht nennen kann, um Entschuldigung. Genannt seien Wolfgang Kehr - mein erster Chef in Freiburg, Altpräsident Horst Kern - mein vorletzter Präsident in Göttingen und - es ist vielleicht überraschend - die Journalisten der Rhein-Neckar-Zeitung in Heidelberg, die sich die Überwindung der scheinbar aussichtslosen Bausituation der Universitätsbibliothek zur eigenen Sache gemacht haben. Mit Thomas Oppermann habe ich eine weitere Person schon erwähnt, der ich besonders viel verdanke. Auch Klaus Saur möchte ich noch nennen, der u. a.

50 Einasto (2015) $254 \mathrm{ff}$.

51 Bonte (2020) 381. die Gründung der Zeitschrift BIBLIOTHEK - Forschung und Praxis sowie die große Mikroficheedition der Druckschriften der Bibliotheca Palatina ermöglicht hat. Ich möchte aber außer ihnen auch all den Vielen danken, die mich oft aufopfernd an vielen Orten bei den unterschiedlichsten Aktivitäten unterstützt haben - auch denen, die dieses Symposium vorbereitet und organisiert haben. Wo ich Erfolge hatte, war es letztlich ihr Werk. Mein besonders herzlicher Dank geht auch hier an meine Frau und meine Kinder.

Die Vielfalt des bibliothekarischen Berufs und der damit verbindbaren Aktivitäten vom Bauen und Managen bis zum Digitalisieren und Forschen haben mir erkennbar viel Freude gemacht. Für mich aber war es das Schönste, wenn ich andere fördern konnte. Die Transformation, sagen wir einfacher, die Entwicklung, die ich bei vielen beobachten konnte, wenn sie an Aufgaben wuchsen und mich dabei oft weit überholten, waren für mich der beste Teil des manchmal auch „lausigen“ Managements von Bibliotheken.

\section{Literaturverzeichnis}

Arbeitsgruppe Bibliotheksplan Baden-Württemberg (Hrsg.) (1973): Gesamtplan für das wissenschaftliche Bibliothekswesen. Unter Mitarbeit von Elmar Mittler (Redaktion). Bd. 1. München: Verlag Dokumentation.

Arbeitsgruppe Bibliotheksplan Baden-Württemberg (Hrsg.) (1975): Gesamtplan für das wissenschaftliche Bibliothekswesen. Unter Mitarbeit von Elmar Mittler (Redaktion). Bd. 2. München: Verlag Dokumentation.

BDB - Bundesvereinigung deutscher Bibliotheksverbände (1993): Bibliotheken '93. Strukturen, Aufgaben, Positionen. Leipziger Fassung. Leipzig.

BDB - Bundesvereinigung Deutscher Bibliotheksverbände (1994): Bibliotheken ‘93. Strukturen - Aufgaben - Positionen. Unter Mitarbeit von Hans-Jürgen Kuhlmeyer und Simone Rennert. 1. Aufl. Berlin, Göttingen: Dt. Bibliotheksinstitut. Verfügbar unter https://bibliotheksportal.de/content/uploads/2017/10/ bibliotheken93.compressed.pdf.

BDB - Bundesvereinigung deutscher Bibliotheksverbände (1996): Bibliotheken im Zeitalter der Datenautobahnen und internationale Netze. In: Deutschlands Weg in die Informationsgesellschaft. Herausforderungen und Perspektiven für Wirtschaft, Wissenschaft, Recht und Politik, hg. v. Jörg Tauss. Unter Mitarbeit von Jörg Tauss, Johannes Kollbeck und Jan Mönikes. 1. Aufl. Baden-Baden: Nomos-Verlagsgesellschaft, 756-62.

Bisbrouck, Marie-Franco̧ise; Mittler, Elmar (Hrsg.) (1997): The postmodern library between functionality and aesthetics. Proceedings of the seminar of the LIBER Architecture Group, Paris, 22-26 January 1996. Proceedings of the seminar of the LIBER Architecture Group. Graz: Akad. Druck- u. Verlags-Anstalt (Special issue of European Research Libraries cooperation). 
Bonte, Achim (2017): Vorstoß in neue Wissensräume. Makerspaces im Leistungsangebot wissenschaftlicher Bibliotheken. In: Strategien für die Bibliothek als Ort. Festschrift für Petra Hauke zum 70. Geburtstag, hg. v. Konrad Umlauf, Klaus Ulrich Werner und Andrea Kaufmann. Unter Mitarbeit von Petra Hauke. Berlin, Boston: De Gruyter Saur, 84-94.

Bonte, Achim (2020): Diversifizierte Forschungsunterstützung statt Pfeifen im Walde - „SLUB 2025“ und die Zukunft der Bibliotheken. In: ZfBB, 67 (5-6), 379-82. DOI: 10.3196/ 18642950206756106.

Bonte, Achim; Muschalek, Antonie (Hrsg.) (2019): SLUB 2025 Wissen teilen - Menschen verbinden. Strategie der Sächsischen Landesbibliothek - Staats- und Universitätsbibliothek Dresden. Dresden. Verfügbar unter https://nbn-resolving.org/urn:nbn:d e:bsz:14-qucosa2-357501.

Börsenvereins des Deutschen Buchhandels; Bundesvereinigung Deutscher Bibliotheksverbände (BDB) (1996): Bibliotheken und Buchhandel vor der online-Herausforderung. Gemeinsame Erklärung des Börsenvereins des Deutschen Buchhandels und der Bundesvereinigung Deutscher Bibliotheksverbände. In: Bibliotheksdienst, 30 (7), 1183-84.

Boyle, Leonard; Mittler, Elmar (Hrsg.) (1989-1995): Bibliotheca Palatina. Druckschriften $=$ Stampati Palatini $=$ Printed books. 21103 Mikrofiches. München: Saur.

Bund-Länder-Arbeitsgruppe Bibliothekswesen (Hrsg.) (1991): Empfehlungen zur Förderung der Bibliotheken in den neuen Bundesländern. Unter Mitarbeit von Michael Hirsch und Antonius Jammers (Hrsg.) und Helmut Rösner (Red.). Berlin: Dt. Bibliotheksinstitut (dbi-Materialien: 106).

Ceynowa, Klaus (2015): Vom Wert des Sammelns und vom Mehrwert des Digitalen. Verstreute Bemerkungen zur gegenwärtigen Lage der Bibliothek. In: BIBLIOTHEK - Forschung und Praxis, 39 (3), 68-276. DOI: 10.1515/bfp-2015-0042.

Cronin, Blaise (1983): Meanwhile in West Germany... In: Aslib Proceedings, 35 (5), 213-21. Verfügbar unter http://dx.doi.org/10.1108 /eb050884.

Degkwitz, Andreas (2016): „I have a dream ...“. Bibliothek der Zukunft. In: Bibliothek der Zukunft - Zukunft der Bibliothek. Festschrift für Elmar Mittler anlässlich seines 75. Geburtstags, hg. v. Andreas Degkwitz. Berlin, Boston: De Gruyter Saur, 24-33.

DFG - Deutsche Forschungsgemeinschaft (1970): Empfehlungen für die Zusammenarbeit zwischen Hochschulbibliothek und Institutsbibliotheken. Bonn-Bad Godesberg.

Dietrich, Viktoria (1984): Erinnerungen an EDV in Australien. In: Theke. Informationsblatt der Mitarbeiter im Bibliothekssystem der Universität Heidelberg, (2), 18-20.

Dorsch, Klaus-Dieter (1985): Gesamtkataloge in Baden-Württemberg. Die lokalen Monographien-Gesamtkataloge im Bezugsnetz mehrschichtiger Hochschulbibliothekssysteme in Baden-Württemberg. Heidelberg: Univ.-Bibliothek Heidelberg (Heidelberger Bibliotheksschriften: 21).

Einasto, Olga (2015): Transforming library communication. From Gutenberg to Zuckerberg. In: New library world, 116 (5/6), 247-63. DOI: 10.1108/NLW-05-2014-0055.

Horstmann, Wolfram (2014): Die Bibliothek als Werkstatt der Wissenschaft. In: BIBLIOTHEK - Forschung und Praxis, 38 (3), 503-05. DOI: 10.1515/bfp-2014-0056.

Kasch, Petra (2008): Der Aufbau öffentlicher Bibliotheken nach 1989 in den neuen Bundesländern. Kultureller Verlust und Suche nach einer neuen Identität? Zugl.: Berlin, Humboldt-Universität,
Masterarbeit. Berlin: Institut für Bibliotheks- und Informationswissenschaft. Humboldt-Universität (Berliner Handreichungen zur Bibliotheks- und Informationswissenschaft: 228). Verfügbar unter http://webdoc.sub.gwdg.de/ebook/serien/aw/Berliner_ Handreichungen/h228.pdf.

Köster, Philipp (2001): Glosse „Elfenbeinturm, 1. Stock“. Der Bibliothekar. In: Spiegel, 29.5.2001. Verfügbar unter https://www.spi egel.de/lebenundlernen/uni/glosse-elfenbeinturm-1-stock-derbibliothekar-a-124532.html.

McDonald, Andrew (2006): The Ten Commandments revisited. The qualities of good library space. In: LIBER Quarterly, 16 (2). Verfügbar unter https://www.liberquarterly.eu/articles/10.1835 2/lq.7840/.

Metcalf, Keyes DeWitt; Ellsworth, Ralph Eugene; Faulkner-Brown, Harry (Hrsg.) (1971): Planning the academic library. Metcalf and Ellsworth at York. Newcastle upon Tyne: Oriel.

Mittler, Elmar (1970): Modelle der Erwerbungskooperation an Universitäten. In: Zeitschrift für Bibliothekswesen und Bibliographie, 17 (4-5), 288-300. Verfügbar unter http://webdoc.sub.gwdg.d e/pub/sub/2007/mitt70-modelle.pdf.

Mittler, Elmar (1972): Moderne Bibliotheksplanung. Ein Überblick über neuere Planungsmethoden und erste Ansätze ihrer Anwendung bei der Bibliotheksplanung in Baden-Württemberg. In: ZfBB, 19 (4-5), 260-84. Verfügbar unter http://webdoc.sub.gwd g.de/pub/sub/2007/mitt72-bibliotheksplanung.pdf.

Mittler, Elmar (1973): Gesamtplan für das wissenschaftliche Bibliothekswesen. 2. Aufl. Pullach bei München: Verlag Dokumentation.

Mittler, Elmar (1976): Bibliotheksplanung. In: Zur Theorie und Praxis des modernen Bibliothekswesens, hg. v. Wolfgang Kehr, Karl Wilhelm Neubauer und Joachim Stoltzenburg. Bd. 1. München: Verl. Dokumentation, 88-154. Verfügbar unter http://webdoc.s ub.gwdg.de/pub/sub/2007/mittbiblp_PPN518909107.pdf.

Mittler, Elmar (1985): Wie bewältigen wir die wachsende Informationsflut. In: Theke. Informationsblatt der Mitarbeiter im Bibliothekssystem der Universität Heidelberg (1), 1-14.

Mittler, Elmar (1986b): Problem erkannt? In: Theke. Informationsblatt der Mitarbeiter im Bibliothekssystem der Universität Heidelberg, (1), 12-17.

Mittler, Elmar (1989): HEIDI. Das Heidelberger Bibliothekssystem als lokales System im regionalen Verbund. In: Verband der Bibliotheken des Landes Nordrhein-Westfalen. Mitteilungsblatt, NF 39 , 256-62.

Mittler, Elmar (1990): Auf dem Weg zur Einheit im deutschen Bibliothekswesen. Die ersten sechs Monate. In: Zeitschrift für Bibliothekswesen und Bibliographie, 37 (4), 354-57. Verfügbar unter $\mathrm{h}$ ttp://webdoc.sub.gwdg.de/pub/sub/2007/mitt90-einheit.pdf.

Mittler, Elmar (1993a): „Bibliotheksplan ‘73“ - „Bibliotheken ‘93“. Zum Stand der Überarbeitung. „Bibliotheksplan '73“ - „Bibliotheken '93“. In: Bibliotheksdienst, 27 (11), 1657-58. Verfügbar unter http://webdoc.sub.gwdg.de/pub/sub/2007/mittbibl73_P PN543709574.pdf.

Mittler, Elmar (1993b): Lasst den Leser an die Bücher. Die Sanierung des Hauptgebäudes der Universitätsbibliothek. In: RupertoCarola, 35 (69), 38-41.

Mittler, Elmar (2001): Vom Bibliothekszentrum für Niedersachsen (BRZN) zur Verbundzentrale des Gemeinsamen Bibliotheksverbundes (VZG). In: Zehn Jahre Pica in Niedersachsen und Deutschland. Skizzen eines Erfolgs, aus Anlass der 5. Verbundkonferenz des Gemeinsamen Bibliotheksverbundes der Länder Bremen, 
Hamburg, Mecklenburg-Vorpommern, Niedersachsen, SachsenAnhalt, Schleswig-Holstein und Thüringen vom 11. - 12. September 2001 in Göttingen, hg. v. Elmar Mittler. Göttingen: Niedersächsische Staats- und Universitätsbibliothek (Göttinger Bibliotheksschriften: 16), 29-46.

Mittler, Elmar (2003): Niedersächsische Staats- und Universitätsbibliothek (SUB Göttingen). In: BIBLIOTHEK - Forschung und Praxis, 27 (1/2), 7-12. Verfügbar unter http://webdoc.sub.gwdg. de/pub/sub/2007/mitt03-bibliotheksbau.pdf.

Mittler, Elmar (2004): The battle for good libraries. In: LIBER Quarterly, 14 (2). DOI: 10.18352/lq.7777.

Mittler, Elmar (2007): Eine Bibliothek für die Welt. Wiedergewinnung der „Bibliotheca Palatina“. In: Ruperto-Carola, (2), 12-17. Verfügbar unter https://www.uni-heidelberg.de/presse/ruca/ru ca07-2/palatina.html.

Mittler, Elmar (2012): Wissenschaftliche Forschung und Publikation im Netz. Neue Herausforderungen für Forscher, Bibliotheken und Verlage. In: Medienkonvergenz - Transdisziplinär, hg. v. Stephan Füssel. Berlin, Boston: De Gruyter (Media convergence: 1), 31-80.

Mittler, Elmar (2013): Die Zeit war reif. Der Aufbau der Abteilung Forschung \& Entwicklung an der SUB Göttingen. In: Evolution der Informationsinfrastruktur. Kooperation zwischen Bibliothek und Wissenschaft, hg. v. Heike Neuroth, Norbert Lossau und Andrea Rapp. Glückstadt: vwh Verlag Werner Hülsbusch Fachverlag für Medientechnik und -wirtschaft.

Mittler, Elmar (2017): Radikaler Wandel? Ein Blick in die Bibliotheksentwicklung der letzten 60 Jahre. In: Bibliothek. Forschung für die Praxis. Festschrift für Konrad Umlaufzum 65. Geburtstag, hg. v. Petra Hauke, Andrea Kaufmann und Vivien Petras. Berlin, Boston: de Gruyter Saur, 604-24.

Mittler, Elmar (2017): Wohin geht die Reise? Bibliothekspolitik am Anfang des 21. Jahrhunderts. In: BIBLIOTHEK - Forschung und Praxis, 41 (2), 213-23. DOI: 10.1515/bfp-2017-003.

Mittler, Elmar (Hrsg.) (1986a): Bibliotheca Palatina. Katalog zur Ausstellung vom 8. Juli bis 2. November 1986, Heiliggeistkirche Heidelberg. Textband, Bildband. Heidelberg (Heidelberger Bibliotheksschriften).

Mittler, Elmar (Hrsg.) (1999): Bibliotheca Palatina. Druckschriften Stampati Palatini - Printed books. Katalog zur MikroficheAusgabe. München: Saur.

Mittler, Elmar; Dörflinger, Gabriele (1988): HEIDI. Das Heidelberger Bibliothekssystem als lokales System im regionalen Verbund. Heidelberg: Univ.-Bibliothek (Heidelberger Bibliotheksschriften: 34).

Neumayr, Philipp (2021): Palatina Superstar. 75 Jahre RNZ: Als die Landesbibliothek der pfälzischen Kurfürsten nach Heidelberg zurückkehrte Ein nie dagewesener Benutzerandrang. In: RheinNeckar-Zeitung, 01.02.2021 (Heidelberg), 7.

Nissen, Martin (2020): Die Sanierung des Südflügels der Universitätsbibliothek Heidelberg (2016-2019). In: BIBLIOTHEK - Forschung und Praxis, 44 (3), 530-36. DOI: 10.1515/bfp-2020-2022.

Nordrhein-Westfalen/Ministerium für Wissenschaft und Forschung (1974): Gesamthochschulen in Nordrhein-Westfalen. Materialien zu Aufbau, Entwicklung und Funktion. Wuppertal. Verfügbar unter https://digital.ub.uni-paderborn.de/ihd/content/struc ture/3261171.

Passig, Kathrin (2013): Die Zukunft des Papierverleihs. In: ZEIT ONLINE. Verfügbar unter https://www.zeit.de/digital/internet/2 013-11/passig-bibliotheken-internet.
Plassmann, Engelbert; Busse, Gisela von; Ernestus, Horst; Seefeldt, Jürgen (1999): Das Bibliothekswesen der Bundesrepublik Deutschland. Ein Handbuch. 3., völlig neubearb. Aufl. des durch Gisela von Busse und Horst Ernestus begr. Werkes. Wiesbaden: Harrassowitz.

Probst, Veit (2018): Digitization at the Heidelberg University Library. The digital Bibliotheca Palatina project. In: Digital Philology, 6 (2), 213-33.

Riemschneider, I.; Stumpf, C.; Weber, G. (1986): ... allein uns fehlt der Glaube! In: Theke. Informationsblatt der Mitarbeiter im Bibliothekssystem der Universität Heidelberg, (2), 18-21.

Schulz, Knud (2015): Die Bibliothek als innovative Bürgerplattform. In: BuB, (4), 206-10.

Shotton, David; Portwin, Katie; Klyne, Graham; Miles, Alistair (2009): Adventures in semantic publishing: exemplar semantic enhancements of a research article. In: PLoS computational biology, 5 (4), e1000361. DOI: 10.1371/journal.pcbi.1000361.

Türk, Ulrike u. a. (1984): Erinnerungen an EDV in Australien - kritisch gesehen. Zu dem Artikel von V. Dietrich in Theke 1984 (2). In: Theke. Informationsblatt der Mitarbeiter im Bibliothekssystem der Universität Heidelberg, (1), 15-18.

Türk, Ulrike; Brose, Peter (1985): Thema verfehlt. In: Theke. Informationsblatt der Mitarbeiter im Bibliothekssystem der Universität Heidelberg, (2/3), 12-17.

Umlauf, Konrad (2008): Bibliotheksplan 1969 und Bibliotheksplan 1973. Anspruch und Realisierung. In: Aufdem Wege in die Informationsgesellschaft. Bibliotheken in den 70er und 80er Jahren des 20. Jahrhunderts, hg. v. Peter Vodosek und Werner Arnold. Wiesbaden: Harrassowitz (Wolfenbütteler Schriften zur Geschichte des Buchwesens: 43), 27-80.

Valenza, Joyce (2008): Library as domestic metaphor. Verfügbar unter http://blogs.slj.com/neverend-ingsearch/2008/08/25/libraryas-domestic-metaphor.

Wille, Jakob (1911): Brief an das Badische Ministerium für Kultus und Unterricht. Vom 5. August 1911. Generallandesarchiv Karlsruhe 235/166. Verfügbar unter http://www.ub.uni-heidelberg.de/ wir/geschichte/wille.html.

Wissenschaftsrat (1964): Wissenschaftliche Bibliotheken. Tübingen: Mohr (Empfehlungen des Wissenschaftsrates zum Ausbau der wissenschaftlichen Einrichtungen: Teil 2).

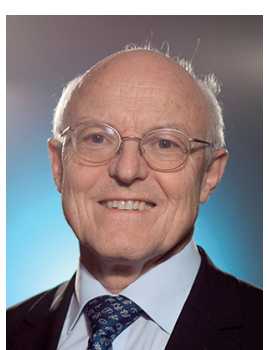

Prof. em. Dr. Drs. h. c. Elmar Mittler Georg-August-Universität Göttingen Niedersächsische Staats- und Universitätsbibliothek Göttingen Papendiek 14 D-37073 Göttingen emittle@gwdg.de 